\title{
Téoros
}

Revue de recherche en tourisme

\section{Certification, accréditation et labellisation en écotourisme}

\section{Maurice Couture}

Volume 21, numéro 3, automne 2002

Écotourisme

URI : https://id.erudit.org/iderudit/1072504ar

DOI : https://doi.org/10.7202/1072504ar

Aller au sommaire du numéro

Éditeur(s)

Université du Québec à Montréal

ISSN

0712-8657 (imprimé)

1923-2705 (numérique)

Découvrir la revue

Citer cet article

Couture, M. (2002). Certification, accréditation et labellisation en écotourisme. Téoros, 21(3), 57-59. https://doi.org/10.7202/1072504ar d'utilisation que vous pouvez consulter en ligne.

https://apropos.erudit.org/fr/usagers/politique-dutilisation/ 


\section{Certification, accréditation et labellisation en écotourisme}

\section{Maurice Coulure}

\section{Contexte général}

C ompte tenu des interprétations très larges qui peuvent être faites des multiples définitions de l'écotourisme et de l'usage de plus en plus répandu du terme, plusieurs intervenants et décideurs considèrent que les produits et les entreprises d'écotourisme devraient faire l'objet d'une évaluation qui confirme leur respect de certaines lignes directrices et de certaines normes préétablies. En bout de ligne, on souhaiterait être en mesure d'affirmer (certifier) que les produits ou les entreprises évalués mettent en pratique les valeurs de l'écotourisme, de les promouvoir autour d'une « marque » commune (label) et d'informer les clientèles de manière appropriée.

\section{Quelques définitions importantes}

Accréditation : Procédé par lequel une organisation est habilitée à certifier des entreprises qui répondent à une norme donnée. À titre d'exemple, le Bureau de normalisation du Québec est une organisation accréditée par divers organismes internationaux ou nationaux (ISO par exemple) pour gérer l'application de diverses normes et procéder à la certification d'entreprises ou de produits.

Certification : Processus par lequel un tiers (organe de certification) assure par écrit au consommateur qu'un produit, un procédé, un service ou un système de gestion respecte des règles précises.

Labellisation : Acte par lequel un organisme affirme que ses membres respectent un règlement qui impose souvent des normes de qualité et certaines exigences professionnelles. Ce processus les associe à une marque (écolabel) et les distingue des autres entreprises du même secteur.
Quelques références sur un sujet au goût du jour

La certification et la labellisation en écotourisme sont devenues des sujets fort populaires. Les séminaires, les conférences, les études et les ouvrages traitant de tels sujets ont grandement proliféré au cours des deux dernières années. À titre indicatif, les plus récents ouvrages sur le sujet sont:

- 2002 : Ecotourism and Certification: Setting Standards in Practice, de Martha Honey ;

- 2001 : Tourism Ecolabelling, Certification and Promotion of Sustainable Management, de Xavier Font et Ralf C. Buckley ;

- 2001 : Protecting Paradise: Certification Programs for Sustainable Tourism and Ecotourism, produit par l'Institute for Policy Studies, Washington, DC ;

- 2000 : Tourism Certification: An Analysis of Green Globe 21 and Other Tourism Certification Programmes, produit par la firme Synergy pour le compte du World Wildlife Fund (WWF) - Royaume-Uni ;

- 2000 : Feasibility and Market Study for an European Eco-Label for Tourist Accomodations, produit par Consultancy and Research for Environmental Management, pour le compte de la Direction générale de l'environnement de la Communauté européenne.

\section{Point de vue des voyageurs}

Plusieurs études tendent à démontrer que la certification et la labellisation en écotourisme sont des services qui ont devancé la demande. La performance environnementale jouerait encore un rôle mineur dans la sélection par les clients d'une destination et d'une entreprise touristiques. Les facteurs décisifs demeurent : qualité des environnements - paysage, forêt, plans d'eau -, santé, sécurité, tranquillité, propreté des lieux. Toutefois, les consommateurs sont de plus en plus ouverts à de tels outils de ré- férence et d'information et conçoivent le label « écologique » comme un outil pratique pour communiquer la performance environnementale d'une entreprise. Un sondage réalisé en 1999 par Tourism Queensland auprès de touristes qui ont consommé des produits accrédités par le Nature and Ecotourism Accreditation Program démontre que :

- $87 \%$ d'entre eux seraient très enclins ou enclins à choisir un produit certifié après avoir reçu des explications sur ce dernier ;

- $37 \%$ des clients interrogés étaient prêts à payer jusqu'à $5 \%$ de plus et $30 \%$ jusqu'à $10 \%$ de plus pour un produit certifié.

\section{Quelques exemples de programmes de certification et d'écolabels}

Les processus de certification ou labels écologiques en tourisme sont particulièrement répandus en Europe, où l'on dénombre plus de quarante sceaux écologiques (écolabels). La multiplication des outils d'information - des « marques » - aurait même pour résultat de créer une certaine confusion auprès des clientèles. Des programmes visant, pour les uns, à certifier les pratiques environnementales ou de tourisme durable des entreprises et, pour les autres, à authentifier les produits d'écotourisme, ont été mis en place un peu partout dans le monde. En voilà quelques exemples :

1 En France, les Gîtes Panda correspondent à une marque développée en partenariat par la WWF-France, la Fédération française des Parcs naturels régionaux (FFPNR) et la Fédération des Gîtes de France (http://www.wwf.be/fr/espa ce/8.html). Cette marque s'appuie notamment sur un réseau de 250 hébergements ruraux, tous situés dans des parcs naturels régionaux et nationaux. La WWF accorde aux Gîtes le droit d'utili- 
ser son logo à certaines conditions (offres d'occasions d'observation de la nature ; souci de préserver l'environnement ; proximité de départs ou de passages de chemins de randonnée).

\section{La CST au Costa Rica (Certificación} para la Sostenibilidad Turística / Certificat pour la soutenabilité touristique) a été conçue de façon à distinguer les entreprises selon leur conformité à un modèle de tourisme durable qui tient compte des interactions entre une entreprise et les ressources naturelles, culturelles et sociales qui l'entourent (http://www.turismo-

sostenible.co.cr/FR/sobreCST/aboutcst.shtml). Ce programme gouvernemental, lié à une stratégie nationale de tourisme durable, répond à plusieurs des principes de l'écotourisme et met en perspective l'importance de l'environnement naturel.

\section{Le Green Tourism Business Scheme} (GTBS) correspond à un processus de certification environnementale en tourisme (http://www.green-business. co.uk/) initié par le Scottish Tourist Board et géré par une de ses divisions, la Quality Assurance. Le programme est considéré, par la plupart des organisations publiques de tourisme d'Écosse, comme une mesure tangible et efficace pour aider les entreprises à s'engager dans des activités à caractère durable. L'évaluation vise différentes catégories d'activités des entreprises : déchets, énergie, eau et eaux usées, transport, fournisseurs et sous-traitants, employés, entreposage, communications et marketing, bruit. Trois différentes accréditations sont possibles : bronze, argent et or.

\section{Le Nature \& Ecotourism Accredita-} tion Program (NEAP) a été lancé en 1996 et a depuis fait l'objet de diverses améliorations. Sa mise en place visait à répondre à un besoin exprimé par les opérateurs d'activités écotouristiques de l'Australie, qui voulaient se différencier de l'ensemble des opérateurs d'activités en milieu naturel (naturebased tourism) et des nombreux «pseudo-produits d'écotourisme » offerts sur le marché. Le NEAP a été principalement développé par l'Ecotourism Association of Australia (EAA); il vise à fournir aux consommateurs et à l'industrie l'assurance que les produits certifiés rencontrent les normes suivantes: bonnes pratiques de gestion environnementale, contenu d'interprétation de qualité et offre d'expériences d'écotourisme de qualité. Ce programme ne vise pas la certification de l'entreprise, mais bien le ou les produits d'écotourisme qu'une entreprise est susceptible d'offrir. La plus récente version (le NEAP II) offre trois différentes certifications : tourisme de nature, écotourisme et écotourisme avancé. Il est prévu que la version III du NEAP fasse un lien avec la reconnaissance professionnelle de guides en écotourisme et en tourisme de nature qui a aussi été initiée par l'Association d'écotourisme de l'Australie, en collaboration avec des institutions de formation.

\section{La norme de produit d'écotourisme du} Programme Qualité de l'industrie touristique du Québec, qui est à l'état de projet (projet de norme P 9700-960) et qui devrait être introduite au cours de 2003, préconise aussi une approche par produit comme en Australie. Cette norme de certification en écotourisme, initiée par Tourisme Québec et élaborée par le Bureau de normalisation du Québec avec la collaboration de l'industrie, se distingue du fait qu'elle se rattache à un programme « qualité » plus « global» qui se réfère à une « norme générale » et à des « normes sectorielles». Quelque onze secteurs sont actuellement concernés et d'autres pourront s'y ajouter : hébergement, pourvoiries, attractions et événements, transport par autobus, croisières-excursions et traversiers, tourisme de nature et aventure, etc. La « norme sectorielle »spécifie les exigences de qualité de la prestation des services à la clientèle offerts par les entreprises d'un secteur donné (norme verticale), tandis que la «norme produit d'écotourisme» certifie la qualité (et l'authenticité) d'un ou de plusieurs produits offerts par une entreprise certifiée (norme horizontale). Parmi les obligations de la « norme produit d'écotourisme » figurent : respect de l'environnement naturel et humain, plan de gestion des impacts sur le milieu naturel, politique de marketing responsable, plan d'interprétation documenté qui s'appuie sur des sources reconnues et des procédures écrites concernant l'observation des animaux, volet éducatif destiné à la clientèle et à son personnel, etc.

Quel que soit le programme, quelques-uns des facteurs de succès concernent : l'intervention d'un organisme tiers, le processus de certification, la mise en place d'un programme qui soit à la fois rigoureux et abordable pour un secteur majoritairement composé de petites entreprises, la né-

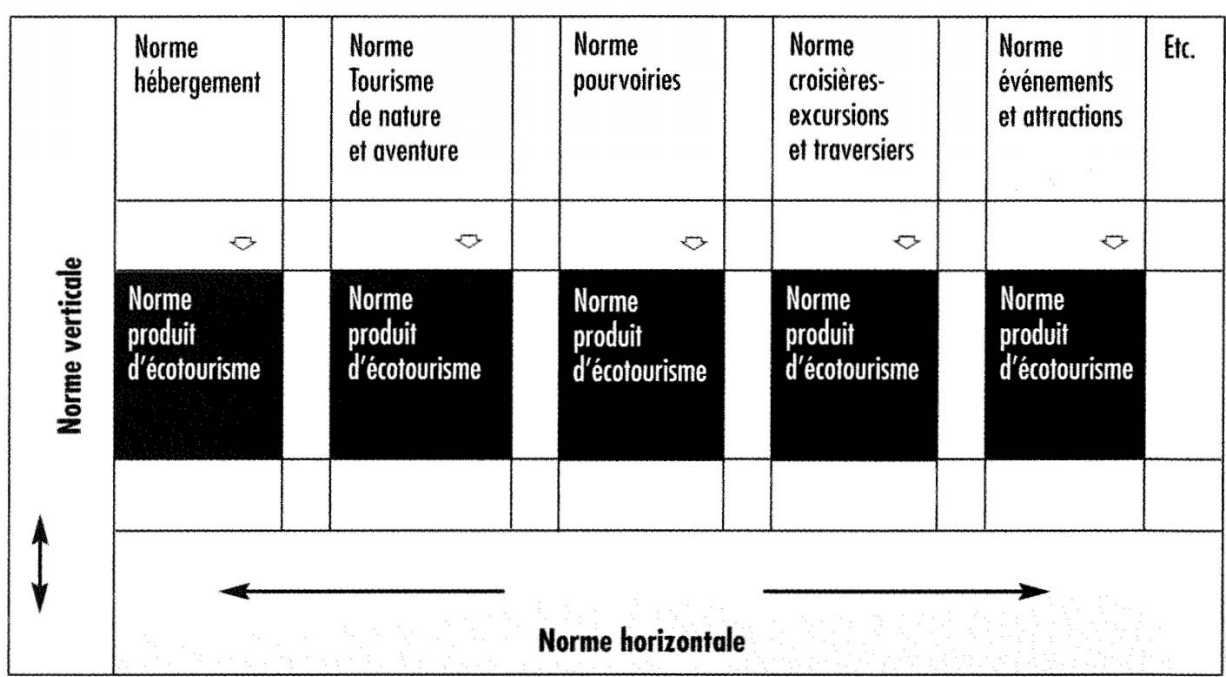




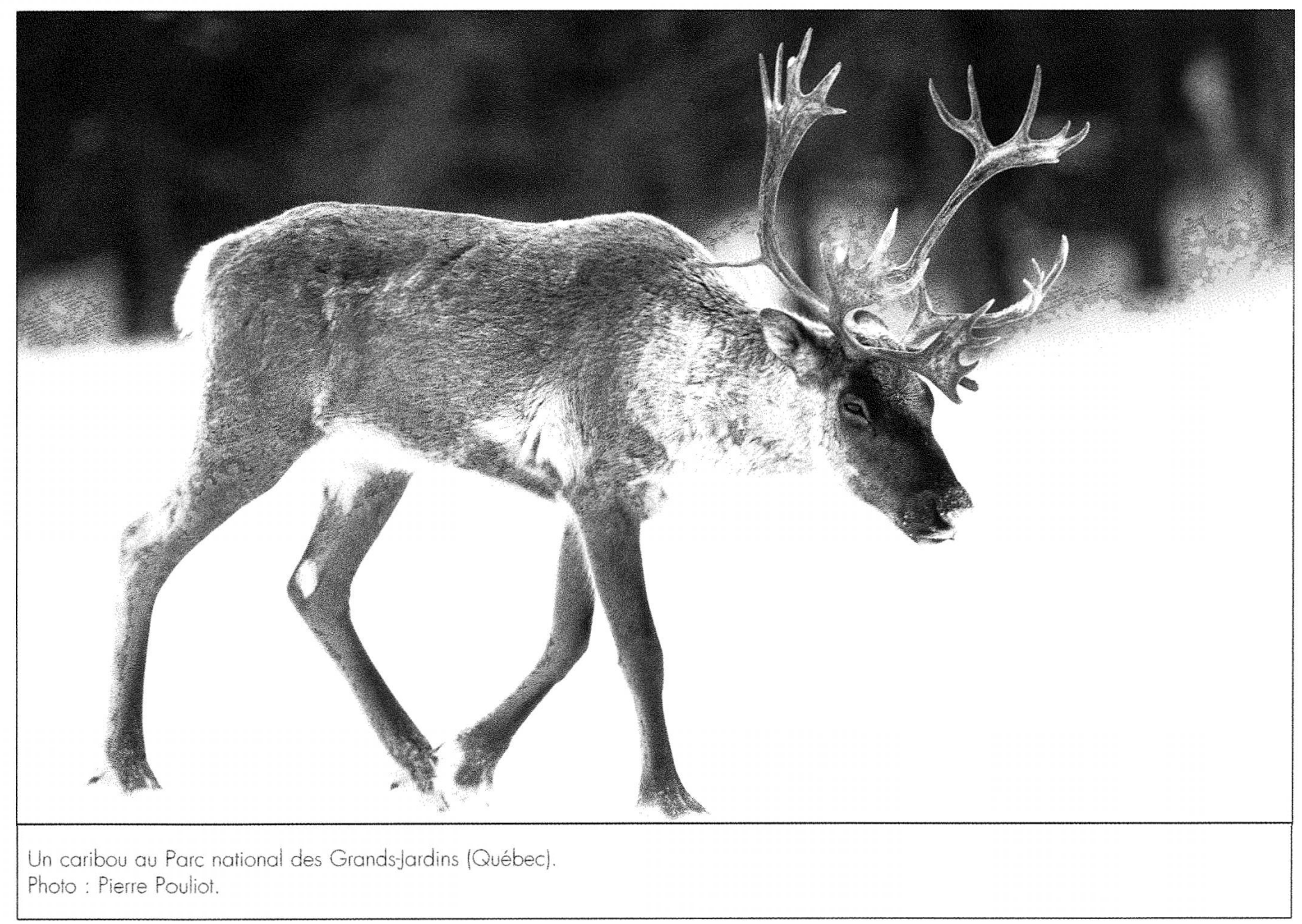

cessité d'adjoindre des avantages et des incitatifs aux entreprises ou aux produits certifiés, notamment sur le plan du marketing, afin de les distinguer des autres entreprises et produits, la constance et le partenariat dans le positionnement de la marque - souvent un long processus auprès des marchés.

\section{Initiatives pour une norme de certification internationale}

Green Globe 21, relié au World Travel and Tourism Council (WTTC), se veut un programme qui vise les pratiques de tourisme durable et qui préconise maintenant une approche de benchmarking. Le bureau chargé de GG21 dans le Sud Pacifique a établi une alliance avec les gestionnaires du programme australien ci-dessus mentionné pour créer le « NEAP international ».

De son côté, la Rain Forest Alliance (http://www.rainforest-alliance.org), or- ganisation internationale de conservation qui a initié le programme de certification «SmartVoyager », préconise une approche d'accréditation des programmes de certification développés par différentes destinations. L'OMT (Organisation mondiale du tourisme) et la Rain Forest Alliance évaluent la faisabilité de créer un Conseil de gestion du tourisme durable (CGTD) qui agirait comme organisme mondial d'agrément dans le secteur du tourisme et qui serait appelé à travailler de concert avec les organismes d'agrément nationaux. Soulignons que le NEAP International viserait des produits d'écotourisme alors que le CGTD aborderait les pratiques de tourisme durable.

Les défis sont nombreux et touchent un bon nombre d'éléments, dont : le degré de support gouvernemental à la certification qui peut influencer énormément les normes des programmes nationaux existants ; $l$ 'in- tégration des marques existantes par rapport à un nouveau logo international de certification ; les différences pouvant exister sur le plan des cultures de gestion face à la qualité et à la capacité de payer des entreprises, notamment entre celle des pays développés et en développement.

Plusieurs recommandations de la Déclaration de Québec sur l'écotourisme font explicitement référence à la mise en place de systèmes de certification et d'écolabels ou d'autres initiatives du genre et insistent sur l'utilisation de lignes directrices reconnues et validées à l'échelle internationale : le développement et l'adoption de normes internationales; l'adoption d'un système de certification fiable qui reflète les critères établis sur les plans régional et local et le caractère volontaire de telles initiatives. 